\title{
Recomendaciones de la Sociedad Mexicana de Nutrición y Endocrinología SMNE ante el COVID-19 para el tratamiento de las personas que viven con DM2
}

\author{
Recommendations of the SMNE facing COVID-19 \\ for the treatment of people living with DM2
}

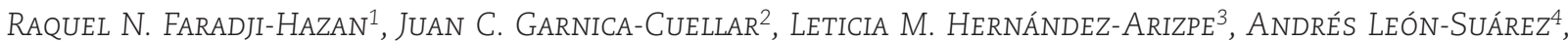

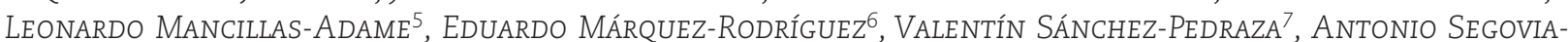

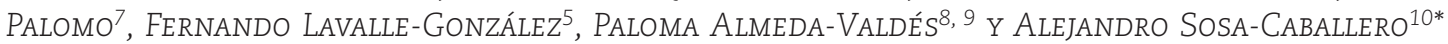

\begin{abstract}
'Dirección, Clínica EnDi, Ciudad de México; ${ }^{2}$ Departamento de Endocrinología, Clínica de Diabetes y Obesidad, Centro Médico Nacional 20 Noviembre, Ciudad de México; ${ }^{3}$ Facultad de Salud Pública y Nutrición, Universidad Autónoma de Nuevo León, Monterrey, N.L.; ${ }^{4}$ Departamento de Endocrinología, Centro Médico ABC, Ciudad de México; ${ }^{5}$ Facultad de Medicina, Servicio de Endocrinología, Hospital Universitario “Dr. José Eleuterio González", Universidad Autónoma de Nuevo León, Monterrey, N.L.; ${ }^{6}$ Dirección Médica, Instituto Jalisciense de Metabolismo, Guadalajara, Jal.; ${ }^{7}$ Departamento de Endocrinología, Hospital General de México, Ciudad de México; ${ }^{8}$ Departamento de Endocrinología, Instituto Nacional de Ciencias Médicas y Nutrición "Salvador Zubirán", Ciudad de México; "Unidad de Investigación de Enfermedades Metabólicas, Instituto Nacional de Ciencias Médicas y Nutrición "Salvador Zubirán", Ciudad de México; ${ }^{10}$ Departamento de Endocrinología, Centro Médico Nacional La Raza, Ciudad de México. México
\end{abstract}

\section{RESUMEN}

La población mexicana con diabetes mellitus se encuentra en mayor riesgo de enfermedad grave y de mortalidad por coronavirus 2 del síndrome respiratorio agudo grave (SARS-CoV-2). Los factores que contribuyen a esto son el potencial mayor riesgo a infecciones, el estado proinflamatorio, procoagulante, la inadecuada regulación inmunológica que sufren las personas con diabetes y el descontrol glucémico, particularmente importante en nuestra población. Por ello, la Sociedad Mexicana de Nutrición y Endocrinología formó un Grupo para Recomendaciones ante la enfermedad por coronavirus 2019 (COVID-19) y emite estas recomendaciones para el manejo terapéutico de la diabetes mellitus tipo 2 con la información actual, mismas que podrán ampliarse o corregirse a la luz de nueva evidencia.

Palabras clave: Diabetes. COVID-19. Recomendaciones. Antidiabéticos.

\begin{abstract}
The Mexican population with diabetes mellitus is at increased risk of severe disease and mortality from severe acute respiratory syndrome coronavirus 2 (SARS-CoV-2). The factors that contribute to this are the potential increased risk of infections, the proinflammatory, procoagulant state, the inadequate immune regulation suffered by people with diabetes and the lack of glycemic control, particularly important in our population. For this reason, the Sociedad Mexicana de Nutrición y Endocrinología formed a group for recommendations facing coronavirus disease 2019 (COVID-19) pandemic and issues these recommendations for the therapeutic management of type 2 diabetes with the current information, which may be expanded or corrected considering new evidence.
\end{abstract}

Key words: Diabetes. COVID-19. Recommendations. Antidiabetics.
Correspondencia:

*Alejandro Sosa-Caballero

E-mail: alexsc_1999@yahoo.com
Fecha de recepción: 11-05-2020

Fecha de aceptación: 25-05-2020

DOI: $10.24875 /$ RME.20000044
Disponible en internet: 12-06-2020

Rev Mex Endocrinol Metab Nutr. 2020;7:122-31

2462-4144 / ( 2020 Sociedad Mexicana de Nutricion y Endocrinologia, AC. Publicado por Permanyer. Éste es un artículo open access bajo la licencia CC BY-NC-ND (http://creativecommons.org/licenses/by-nc-nd/4.0/). 


\section{INTRODUCCIÓN}

Del 20 al 50\% de las personas que tienen la enfermedad por coronavirus 2019 (COVID-19) presentan hiperglucemia en algún momento de la infección por coronavirus 2 del síndrome respiratorio agudo grave (SARS-CoV-2) ${ }^{1}$.

El $10 \%$ de las personas con diabetes presentan la forma grave y mayor riesgo de mortalidad ${ }^{2,3}$; los factores que contribuyen a esto son el potencial mayor riesgo de padecer infecciones ${ }^{4}$ y el descontrol glucémico.

Se ha demostrado que con hemoglobina glucosilada A1c (HbA1c) > 9\% aumenta la posibilidad de neumonía grave ${ }^{5}$. En tanto la mortalidad en este grupo de riesgo va del 22 al $31 \%{ }^{2,6-8}$.

Otros factores descritos que pueden contribuir a estos desenlaces son: el incremento en la replicación viral en células del epitelio pulmonar expuestas a altas concentraciones de glucosa, como se ha reporta en estudios in vitro ${ }^{9}$; la alteración de la mecánica pulmonar con reducción de la capacidad vital forzada en hiperglucemia ${ }^{9}$; el estado proinflamatorio, procoagulante y la inadecuada regulación del sistema inmunitario ${ }^{9-12}$, que se relacionan con la sobreexpresión de citocinas y tormentas inflamatorias ${ }^{13-15}$.

La población mexicana con diabetes mellitus se encuentra en mayor riesgo debido a que se estima que solo el $25 \%$ se encuentran en metas de control de HbA1c y a la baja frecuencia de realización de esta prueba, que solo llega al $15 \%$ de las personas una vez al año ${ }^{16}$.

Por el momento no hay evidencia robusta con relación al tratamiento más adecuado en pacientes con diabetes infectados por SARS-CoV-2. El control de la glucemia debe sostenerse en los objetivos de control, la presencia de comorbilidades, complicaciones preexistentes asociadas a la diabetes y a la consideración de interacciones farmacológicas ${ }^{9}$.

Todos estos factores sustentan la importancia de establecer la eficacia y seguridad de la terapia antidiabética en distintos escenarios, como situaciones sin infección, presencia de COVID-19 de manejo ambulatorio y aquella que requiere hospitalización por la severidad del cuadro clínico ${ }^{17}$.

La Sociedad Mexicana de Nutrición y Endocrinología (SMNE) formó un grupo para recomendaciones ante la COVID-19 y emite estas recomendaciones con la evidencia actual, misma que podrá requerir ampliarse o corregirse a la luz de nueva evidencia.

\section{MANEJO TERAPÉUTICO DE LA DIABETES MELLITUS ANTE LA COVID-19}

Para las personas que viven con diabetes sin COVID-19, se recomienda mantener el tratamiento siempre y cuando se encuentre con adecuado control para enfrentar al SARS-CoV-2 en caso de contraerlo; de lo contrario debe ajustarse el tratamiento para lograr las metas de control.

Existen diferentes familias terapéuticas para el control de la diabetes tipo 2, incluyendo terapias orales (biguanidas, sulfonilurea, tiazolidinedionas [TZD], inhibidores de la dipeptidil peptidasa [iDPP4] e inhibidores del cotransportador sodio-glucosa tipo 2 [iSGLT-2]) e inyectables como los agonistas del receptor del péptido similar al glucagón tipo 1 (GLP-1) y la insulina ${ }^{18}$.

\section{Recomendación 1. Metas de control glucémico}

- Para una persona con diabetes mellitus tipo 2 en control, sin tratamiento con insulina, es aceptable realizar automonitoreo de glucosa capilar en ayuno y posprandial dos o tres veces por semana ${ }^{10}$.

- En personas con diabetes tipo 2 sin infección, las metas de control son: glucosa de ayuno entre 80 y $130 \mathrm{mg} / \mathrm{dl}$ y dos horas posprandiales $<180 \mathrm{mg} / \mathrm{dl}^{19}$.

- En pacientes con COVID-19 de manejo en casa los objetivos de glucemia en ayuno son entre 80 y $130 \mathrm{mg} / \mathrm{dl}$ y posprandial $<180 \mathrm{mg} / \mathrm{dl}$ a las dos ho$\operatorname{ras}^{19}$.

- Para adultos mayores con COVID-19 que requiere hospitalización o aquellos que están en tratamiento 
con esteroides se recomienda glucosa en ayuno entre 110 y $140 \mathrm{mg} / \mathrm{dl}$ y posprandial $<180 \mathrm{mg} / \mathrm{dl}^{19}$.

- En pacientes graves o en estado crítico con soporte ventilatorio, se recomienda mantener glucosa entre $140-180 \mathrm{mg} / \mathrm{dl}$ para evitar complicaciones. No hay evidencia para recomendar un control más estricto en este grupo de pacientes ${ }^{19}$.

\section{MANEJO FARMACOLÓGICO DE LA} DIABETES TIPO 2. BIGUANIDA

La metformina aparece como de primera línea en la mayoría de las guías. Sus beneficios son amplios: potencia antihiperglucémica, mínimo riesgo de hipoglucemia y mejoría de la sensibilidad a la insulina, entre otras.

Sin embargo, en esta coyuntura es preciso determinar el impacto que el uso de la metformina tendría ante el contagio por esta nueva variante de coronavirus. No existe aún evidencia directa sobre la COVID-19 y el tratamiento con metformina, sin embargo algunas experiencias previas con otros procesos virales respiratorios, respuesta en inflamación y citocinas o en daño vascular y metabólico podrían orientar respuestas a esos cuestionamientos.

En ensayos in vitro la metformina ha demostrado consistentemente ser un agente antiproliferativo e inmunomodulador, por medio de la inhibición de la proteincinasa activada por monofosfato de adenina ${ }^{12}$. Diversos estudios han demostrado que la metformina posee efectos antiinflamatorios, con reducción de la activación de la vía del factor nuclear kappa B (NF-к $\beta$ ) y consecuentemente una reducción de las citocinas inflamatorias ${ }^{20}$. La vía del NF-kB está ligada a la orquestación, modulación y adaptación inmunitaria ${ }^{21}$.

La reducción de la actividad de la interleucina (IL) 6 asociada con metformina es quizá la que podría tener un potencial impacto en la COVID-19, dado el gran cúmulo de trabajos que tienen como centro de análisis a esta IL, tanto en la tormenta de citocinas como en la falla orgánica múltiple que genera los casos más graves de la infección por SARS-CoV-222.

En contrapunto, algunos reportes sugieren que los efectos inmunomoduladores de la metformina podrían minar la respuesta ante una neumonía grave $^{23}$.

Finalmente, un reporte preliminar y que requiere aún evaluación reporta toxicidad potencial al combinar hidroxicloroquina o cloroquina y metformina en ratones, pero sin reportes en el campo clínico ${ }^{24}$.

Con los elementos resumidos en las líneas previas, podemos sugerir, con respecto al uso de metformina en diabetes durante a la epidemia por SARSCoV-2, las siguientes consideraciones.

\section{Recomendación 2. Con respecto al uso de metformina}

- En el escenario de diabetes sin COVID-19, no suspender metformina y ajustar según las características individuales del caso.

- En la situación de COVID-19 de manejo en casa, se recomienda vigilancia estrecha, monitoreo y continuar el tratamiento con metformina.

- En el escenario hospitalario o indicios de neumonía por COVID-19, suspender metformina y usar insulina según protocolo.

\section{SULFONILUREAS}

Las sulfonilureas no presentan ninguna interacción con la enzima convertidora de angiotensina II (ECA2) o con metaloproteasa 17 de la familia ADAM $\left(\right.$ ADAM-17) ${ }^{25}$. Sin embargo, aumentan el riesgo de hipoglucemia y deben evitarse en pacientes hospitalizados con enfermedad grave ${ }^{26}$.

Esta es una consideración importante, ya que en algunos estudios se ha reportado que la hidroxicloroquina mejora el control glucémico en pacientes descompensados, refractarios al tratamiento con diabetes; sin embargo, el mecanismo subyacente del efecto hipoglucémico de la hidroxicloroquina sigue sin estar claro. Se ha identificado una reducción en la degradación de la insulina intracelular y el aumento de la acumulación de insulina como 
posibles efectos de la hidroxicloroquina en modelos animales. Por lo tanto, podría ser necesario un ajuste de la dosis de los medicamentos antidiabéticos que causan hipoglucemia, como sulfonilureas o insulina ${ }^{9}$.

RECOMENDACIÓN 3. CON RESPECTO AL USO DE SULFONILUREAS

- En el escenario de diabetes sin COVID-19, no suspender sulfonilurea y ajustar según las características individuales del caso.

- En la situación de COVID-19 de manejo en casa, mantener sulfonilurea con vigilancia más estrecha de la glucemia capilar.

- En el escenario hospitalario o indicios de afección de vías respiratorias bajas por COVID-19, suspender sulfonilurea y usar insulina según protocolo.

INHIBIDORES DE LA DIPEPTIDIL PEPTIDASA TIPO 4

Aunque los iDPP4 se asocian con incremento del riesgo de infecciones nasofaríngeas, no se han relacionado con neumonía ${ }^{27}$.

Se ha identificado que la DPP4 humana, funciona como un receptor para la punta proteica del coronavirus causante del síndrome respiratorio de Oriente Medio (MERS-CoV), pero no para el SARS-CoV- $2^{28}$.

Este ligando interactúa con las células $T$ y factores nucleares, lo que desencadena la cascada inflamatoria. La DPP4 es ubicua y aunque no se conocen todas sus actividades, su involucramiento en el metabolismo de la glucosa, en la inflamación y la inmunidad tienen profundo sustento. La DPP4 está sobreexpresada en la obesidad visceral y correlaciona con inflamación visceral y resistencia a la insulina ${ }^{29}$.

También participa en la sobreexpresión de CD 86 y NF-kB, así como en la activación de las células T. Diversos estudios en humanos han mostrado efectos antiinflamatorios de los iDPP4 y los análogos del receptor de GLP-1, con reducción de la infiltración de macrófagos, disminución de la resistencia a la insulina e inflamación por regulación M1/M2; la alteración en la polarización de los macrófagos también ha sido descrita con los iDPP4 y los miméticos de GLP-130.

Kulcsar, et al. utilizaron modelos de ratones transgénicos diabéticos tipo 2, que sobreexpresan el receptor DPP4 en células alveolares pulmonares para estudiar la gravedad de la infección por el MERS-CoV, demostrando que en estos es más duradera y de peor pronóstico. Ante ello, asumen que el bloqueo del receptor podría determinar un efecto protector $^{30}$.

Por supuesto, se necesita más investigación sobre ese efecto potencialmente protector y su traslación a escenarios clínicos, especialmente en vista de los posibles beneficios terapéuticos esperados de los iDPP4 en personas con diabetes mellitus tipo 2 infectadas por SARS-CoV-2 ${ }^{31}$.

No obstante, ante la evidencia en control glucémico, seguridad cardiovascular, mínimo riesgo de hipoglucemia y los potenciales beneficios a nivel inflamatorio, los iDPP4 emergen como una de las familias terapéuticas de elección en diabetes mellitus tipo 2 durante la epidemia por SARS-CoV- $2^{31}$.

Recomendación 4. Con respecto a los inhibidores de la dipeptidil peptidasa tipo 4

- En el escenario de diabetes sin COVID-19, no suspender iDPP4 y ajustar según las características individuales del caso.

- En la situación de COVID-19 de presentación leve a moderada, vigilancia estrecha, monitoreo y mantener el tratamiento con iDPP4.

- En el escenario hospitalario o indicios de afección de vías respiratorias bajas por COVID-19, usar insulina según protocolo.

- No existe evidencia, pero consideramos podría continuarse el iDPP4 por el potencial beneficio aun en condiciones de hospitalización, bajo consentimiento informado. 


\section{TIAZOLIDINEDIONAS}

En un estudio las TZD incrementaron el riesgo de neumonía en comparación con las sulfonilureas, pero la experiencia clínica es limitada 32 .

Se ha demostrado que la pioglitazona regula la ECA2 en los tejidos sensibles a la insulina en ratas y reduce la actividad de la ADAM-17 en el músculo esquelético en humanos ${ }^{25}$.

Estudios experimentales sugieren que la pioglitazona reduce la esteatohepatitis al aumentar la expresión de ECA2 en el tejido hepático. Este supuesto aumento de la expresión de ECA2 y su relación con la COVID-19 ha llevado a algunos investigadores a proponer evitar la TZD en pacientes con diabetes y COVID-19, pero no se ha demostrado beneficio de suspender la terapia ${ }^{2,32,33}$.

\section{Recomendación 5. Con respecto al uso de tiazolidinedionas}

- En el escenario de diabetes sin COVID-19, no suspender TZD y ajustar según las características individuales del caso.

- En la situación de COVID-19 de manejo en casa, vigilancia estrecha, monitoreo y continuar tratamiento con TZD, en caso de preferencia del médico cambiar por otro antidiabético.

- En el escenario hospitalario o indicios de afección de vías respiratorias bajas por COVID-19, suspender TZD y usar insulina según protocolo.

AGONISTAS DEL RECEPTOR DEL PÉPTIDO SIMILAR AL GLUCAGÓN TIPO 1

Los agonistas del receptor del GLP-1 son otra de las familias de tratamientos que actúan a diversos niveles para mejorar el control de la glucosa y que se asocian con un bajo riesgo de ocasionar hipoglucemia por sí mismos.

En México, los agonistas del receptor de GLP-1 disponibles son inyectables y la frecuencia de su administración y dosificación son variables, siendo los más representativos: lixisenatida, liraglutida, dulaglutida, exenatida y semaglutida. En el caso de la lixisenatida, se encuentra en combinación con un análogo de insulina de acción prolongada (insulina glargina). Los análogos de GLP-1 se han asociado con efectos neutros (exenatida y lixisenatida) o benéficos en cuanto a su impacto en el riesgo cardiovascular (dulaglutida, liraglutida y semaglutida), se asocian todos ellos con reducción de la $\mathrm{HbA} 1 \mathrm{c}$ y con efectos en el peso que van desde neutro hasta reducción, teniendo incluso (a dosis diferentes de las utilizadas en diabetes) indicación o estudios corriendo actualmente para tener indicación en el manejo de obesidad, que es otra comorbilidad asociada a mayor severidad de la COVID-1934.

No existe en este momento una contraindicación por evidencia científica para suspender la utilización de agonistas del receptor de GLP-1 y se pueden utilizar en combinación con insulina. En el paciente que vive con diabetes, cualquier infección puede desencadenar una respuesta inflamatoria que incremente la respuesta metabólica al estrés; en el caso que nos ocupa, la enfermedad relacionada con SARS-CoV-2 puede producir enfermedad que se asocie con descontrol metabólico, por tanto, incrementar la frecuencia de monitoreo de la glucosa puede ser necesario ${ }^{35}$.

Las metas de tratamiento deben ser individualizadas y el paciente deberá estar en comunicación con el personal de salud para identificar la necesidad de intensificar el tratamiento, ajustando la dosis del tratamiento establecido o añadiendo fármacos ${ }^{23}$.

Recomendación 6. Con respecto al uso de agonistas del péptido similar al glucagón tipo 1

- En el escenario de diabetes sin COVID-19, no suspender el tratamiento con análogo de GLP-1 y ajustar según las características individuales del caso.

- En la situación de COVID-19 de manejo en casa, vigilancia estrecha, monitoreo y persistencia de tratamiento con análogo de GLP-1 solo o en combinación con insulina. 
- En el escenario hospitalario por severidad o afección de vías respiratorias bajas por COVID-19, usar insulina según protocolo.

- No existe evidencia, pero consideramos valorar si es necesario la continuidad de análogo de GLP-1 por el potencial beneficio aun en condiciones de hospitalización bajo consentimiento informado.

INHIBIDORES DEL COTRANSPORTADOR SODIO-GLUCOSA TIPO 2

Si bien es cierto que en pacientes con COVID-19 y diabetes no existen al momento ensayos clínicos controlados del manejo de la glucemia con las diversas clases terapéuticas, han surgido evidencias indirectas que harían preferir algunos tratamientos sobre otros. De forma general, los iSGLT-2 han recibido la recomendación (con baja calidad de evidencia) en Reino Unido de no ser utilizados en pacientes que se someterán a tratamiento quirúrgico ni pacientes críticamente enfermos, por la posibilidad de desarrollar cetoacidosis diabética con mayor facilidad ${ }^{36}$.

El control glucémico es importante en cualquier paciente que tenga COVID-19. La mayoría de los pacientes con infección asintomática o leve y con ingesta por vía oral pueden continuar con los medicamentos antidiabéticos habituales. Sin embargo, puede valorarse interrumpir los iSGLT-2 debido al riesgo de deshidratación y cetosis en pacientes con resultado positivo para COVID-1932.

Existe poca evidencia en relación con el metabolismo de la glucosa y el riesgo de cetoacidosis en pacientes con diabetes tipo 2 e infección por COVID-199.

Aunque los iSGLT-2 son generalmente bien tolerados en el entorno ambulatorio, y cardioprotectores sobre todo en el contexto de la insuficiencia cardiaca, la infección por SARS-CoV-2 puede estar asociada con anorexia, deshidratación y deterioro rápido del estado clínico ${ }^{26}$.

La infección por SARS-CoV-2 en las personas con diabetes desencadena una mayor liberación de hormonas como glucocorticoides y catecolaminas, lo que conduce a un incremento en las concentraciones de glucosa?
Por lo tanto, individuos sintomáticos con diabetes tipo 2 e infección activa por SARS-CoV-2 pueden tener mayor riesgo de pérdida de volumen y cetoacidosis euglucémica. En consecuencia, la evidencia disponible sugiere una reevaluación o interrupción de esta clase terapéutica en individuos ambulatorios mal controlados y los iSGLT-2 deben interrumpirse en pacientes inestables con infección grave por SARSCoV-2 al momento del ingreso en el hospital ${ }^{26}$.

Recientemente el Royal United Hospital Bath NHS Foundation Trust publicó que existe un mayor riesgo de cetoacidosis en pacientes con diabetes que se contagian con el virus SARS-CoV-2, por lo que adoptamos las siguientes recomendaciones ${ }^{36}$.

\section{Recomendación 7. Con respecto al uso de inhibidores del cotransportador sodio-glucosa tipo 2}

- A los pacientes con diabetes tipo 1 en tratamiento con iSGLT-2 se les debe aconsejar interrumpir este tratamiento y ajustar las dosis de insulina.

- Aquellos pacientes con diabetes tipo 2 en tratamiento con insulina que han tenido experiencias previas de cetoacidosis en situaciones de enfermedad deben interrumpir el tratamiento con iSGLT-2.

- Los pacientes con diabetes tipo 2 y antidiabéticos orales pueden continuar con el tratamiento con iSGLT-2 siempre y cuando no tengan síntomas de COVID-19, en cuyo caso se puede considerar interrumpirlos.

- En individuos sin diabetes (o con prediabetes) que tienen un alto riesgo cardiovascular y a los que se les prescribió iSGLT-2 deben interrumpir el tratamiento.

- A ningún paciente ingresado por sintomatología de COVID-19 se le debe prescribir un iSGLT-2.

RECOMENDACIÓN 8. CON RESPECTO AL USO DE INSULINA

- En caso de estar bajo tratamiento con insulina, se recomienda mantener su tratamiento y hacer ajustes en la dosis en caso de estar fuera de meta. 
- Para manejo hospitalario, consultar la sección correspondiente.

\section{EDUCACIÓN NUTRIMENTAL EN} DIABETES MELLITUS

Parte del manejo integral de las personas con diabetes mellitus es el manejo de alimentos en escenarios sin COVID-19 o con manifestaciones leves a moderadas de manejo ambulatorio ${ }^{19}$.

\section{Recomendación 9. con respecto al manejo nutricional}

- La persona que vive con diabetes debe planificar menús evitando comprar alimentos hipercalóricos y ultraprocesados.

- Indicar sustituir refrescos azucarados por agua, café, infusiones o tés. Elegir frutos secos, carnes magras, pescado, huevos y lácteos, cereales integrales y no procesados.

- Comer diariamente como mínimo cinco grupos de verduras y dos a tres frutas. Incluir colaciones saludables como: tostadas de maíz con queso fresco, yogurt natural con frutos rojos, verduras, semillas.

- Desinfectar y prepara adecuadamente los alimentos.

- Establecer horarios regulares de alimentación.

- Si el paciente tiene alguna duda, debe contactar un nutriólogo.

\section{CONTACTO CON SU MÉDICO}

En caso necesario, el paciente debe consultar a su médico, para lo cual puede considerar el uso de telemedicina ${ }^{32}$.

\section{Recomendación 10. Con respecto al contacto con el médico}

- Prefiera un método de consulta a distancia electrónico de ser posible.
- El paciente debe ser informado de que en caso de síntomas de COVID-19 de gravedad (disnea, dolor torácico) debe acudir a servicio médico local.

- Contar con una red de apoyo (amigo o familiar).

RIESGOS CON LAS TERAPIAS PARA LA COVID-19 EN LAS PERSONAS CON DIABETES MELLITUS

- Hipoglucemia en pacientes con diabetes mellitus ante COVID-19y su tratamiento. Se ha reportado en el $10 \%$ de las personas con diabetes y COVID- $19^{35}$.

- El uso de cloroquina o de hidroxicloroquina tiene beneficios en las personas con diabetes por sus efectos inmunomoduladores y antiinflamatorios, así como en la producción de péptido $C$, lo que se relaciona con protección de la célula beta de los islotes de Langerhans. Sin embargo, uno de sus riesgos es el desarrollo de hipoglucemia?.

- El uso de esteroides causa hiperglucemia en más del $80 \%$ de las personas con diabetes e incluso en personas sin diabetes previa, lo que en sí mismo constituye un riesgo para la mortalidad. En el caso de requerir tratamiento con estos medicamentos se debe procurar la euglucemia con un monitoreo estrecho con glucemias capilares, lo cual también constituye un dilema por la mayor exposición al SARS-CoV- $2^{8}$.

MANEJO DE LA DIABETES MELLITUS DURANTE LA HOSPITALIZACIÓN POR COVID-19

En todos los pacientes hospitalizados, tanto la hipoglucemia como la hiperglucemia son factores asociados con aumento de morbimortalidad. El manejo debe estar enfocado en la prevención de estas complicaciones agudas para evitar desenlaces desfavorables.

En la pandemia actual, entre el 10 y el $33 \%$ de los pacientes hospitalizados tienen diabetes mellitus 
como comorbilidad, con mayor prevalencia de insuficiencia cardiaca y respiratoria en comparación a los pacientes que no la tienen.

\section{Recomendación 11. Con respecto al paciente hospitalizado}

- Se recomienda que a todos los pacientes con diagnóstico previo de diabetes que sean hospitalizados se les tome una $\mathrm{HbA} 1 \mathrm{c}$ si está disponible en el centro y si no ha sido evaluada en los últimos tres meses ${ }^{37}$.

- Para personas hospitalizadas un esquema basalbolos de insulina es lo más recomendable ${ }^{10}$.

- En casos graves de COVID-19 se recomienda tratamiento con insulina por vía intravenosa ${ }^{10}$.

Se ha visto que los pacientes con infecciones pulmonares por virus producen una reacción inflamatoria extensa que aumenta las lesiones pulmonares y confiere un peor pronóstico al paciente; uno de los factores que la aumenta es la variabilidad glucémica durante la hospitalización, por lo que se debe intentar disminuir mediante el monitoreo de glucosa.

\section{RECOMENDACIÓN 12. CON RESPECTO AL MONITOREO DE LA GLUCOSA CAPILAR}

- Los pacientes hospitalizados deben tener monitoreo de glucosa. Se recomienda que en los pacientes que conserven la vía oral conservada o con nutrición enteral se realice medición de glucosa preprandial y antes de dormir.

- En los pacientes con nutrición parenteral o en ayuno se debe medir la glucosa al menos cada 4-6 horas en el contexto de un paciente críticamente enfermo.

- Si el paciente está con infusión de insulina se debe monitorizar cada 30 minutos a 2 horas $^{37}$.

Recientemente la Food and Drug Administration autorizó el monitoreo continuo de glucosa con dispositivos remotos en los pacientes hospitalizados con
COVID-19 para disminuir al máximo el contacto con los enfermos y limitar el riesgo de contagio del personal de salud ${ }^{38}$.

\section{Recomendación 13. Con respecto al uso de monitores continuos de glucosa}

- Se recomienda el uso de monitores continuos de glucosa, si se tienen disponibles. Se debe tomar en cuenta la interacción de estos dispositivos con medicamentos como paracetamol, atenolol o lisinopril, para interpretar los datos de forma adecuada ${ }^{38}$.

- Se recomienda suspender los medicamentos orales y hacer el manejo de los pacientes con insulina subcutánea o intravenosa, de acuerdo con el contexto clínico.

- No hay evidencia suficiente para recomendar otro tratamiento oral $\mathrm{y} / \mathrm{o}$ inyectado adyuvante para el control de la glucosa en este grupo de pacientes.

- El tratamiento con insulina debe ser inicialmente con insulina basal y rápida subcutánea para controlar picos de hiperglucemia y evitar hipoglucemias.

- Si hay hiperglucemia persistente, se puede utilizar una infusión intravenosa de insulina para corregirla ${ }^{39}$.

Una proporción de los pacientes hospitalizados pueden requerir tratamiento con glucocorticoides durante la hospitalización.

RECOMENDACIÓN 14. CON RESPECTO AL MANEJO DE LA HIPERGLUCEMIA EN PACIENTES EN TRATAMIENTO CON ESTEROIDES

- Si se utilizan glucocorticoides de corta acción (p. ej., prednisona oral) lo ideal es monitorizar la glucosa y utilizar insulina intermedia (NPH) e insulina rápida para la corrección de la hiperglucemia posprandial.

- Cuando se utilizan esteroides de larga duración, en múltiples dosis o altas dosis (dexametasona, 
metilprednisolona), se recomienda utilizar insulina basal de larga duración (glargina, degludec) en conjunto con insulina prandial rápida y de corrección de acuerdo con el monitoreo de glucosa ${ }^{9,26,31}$.

- Los pacientes que utilizaban microinfusora de insulina para el tratamiento previo a su ingreso muchas veces pueden continuar con su tratamiento con esta, durante una hospitalización en condiciones no severas.

Un paciente hospitalizado por COVID-19 y diabetes tiene distintos factores para contraindicar este manejo: medicamentos que descontrolan la glucosa como glucocorticoides, riesgo de complicaciones súbitas y necesidad de manejo multidisciplinario, entre otras.

RECOMENDACIÓN 15. CON RESPECTO A LOS PACIENTES QUE USAN MICROINFUSORAS DE INSULINA

- Se recomienda, en la medida de lo posible, mantener la microinfusora con la supervisión de un especialista capacitado, antes de considerar retirarla.

- En caso de que el paciente este inconsciente, no haya algún especialista disponible o tenga criterios de cetoacidosis diabética o estado hiperosmolar hiperglucémico, se recomienda desconectar la microinfusora e iniciar tratamiento de acuerdo con lo recomendado en cada situación clínica. Posiblemente requiera infusión intravenosa de insulina durante la fase aguda.

- Se están realizando estudios sobre el uso de este tipo de dispositivos en pacientes críticos hospitalizados por COVID-19, por el momento no hay datos suficientes para recomendar o no su uso en este contexto $9,26,31$.

RECOMENDACIÓN 16. CON RESPECTO AL RIESGO Y MANEJO DE LA HIPOGLUCEMIA

- La hipoglucemia $(<70 \mathrm{mg} / \mathrm{dl})$ debe tratarse de acuerdo con el mismo protocolo para todos los pacientes hospitalizados en cada centro hospitalario. Se puede utilizar solución glucosada al 50\% 25-50 ml intravenosa en bolo valorando la respuesta en 15 minutos, en pacientes que no tengan nutrición enteral o tengan alterado el estado de alerta.

- En caso de que el paciente este consciente, cooperador y tenga libre la vía oral o nutrición enteral se debe dar $15 \mathrm{~g}$ de carbohidratos y valorar a los 15 minutos y repetir hasta que la glucosa sea $>100$ $\mathrm{mg} / \mathrm{dl}$. Posterior al episodio de hipoglucemia se debe ajustar la dosis de insulina para prevenir nuevos eventos ${ }^{9,26,31}$.

- Se ha observado que la hidroxicloroquina/cloroquina, por un mecanismo aún no muy claro, disminuye los niveles de glucosa en pacientes hospitalizados con COVID-19, por lo que se debe tomar en cuenta para ajustar el tratamiento y evitar hipoglucemias con su uso 9,35 .

\section{CONCLUSIONES}

No hay evidencia robusta en humanos en el campo de la COVID-19 y los antidiabéticos. Por lo tanto, en ausencia de pruebas sólidas, sería precipitado considerar un medicamento sobre el otro, salvo en circunstancias especiales, pero sí la suspensión de los iSGLT-2 como medida de seguridad en el paciente hospitalizado o con antecedente de cetoacidosis.

Un adecuado control glucémico debe ser la meta en toda persona que vive con diabetes mellitus siempre y máxime en un estado de epidemia como la actual, por lo que se proponen estas recomendaciones con el fin de disminuir los riesgos y los efectos de la COVID-19.

\section{FINANCIAMIENTO}

La presente investigación no ha recibido ayudas específicas provenientes de agencias del sector público, sector comercial o entidades sin ánimo de lucro. 
CONFLICTO DE INTERESES

Los autores declaran no tener conflicto de intereses.

\section{RESPONSABILIDADES ÉTICAS}

Protección de personas y animales. Los autores declaran que para esta investigación no se han realizado experimentos en seres humanos ni en animales.

Confidencialidad de los datos. Los autores declaran que han seguido los protocolos de su centro de trabajo sobre la publicación de datos de pacientes.

Derecho a la privacidad y consentimiento informado. Los autores declaran que en este artículo no aparecen datos de pacientes.

\section{BIBLIOGRAFÍA}

1. Chen N, Zhou M, Dong X, Qu J, Gong F, Han Y, et al. Epidemiological and clinical characteristics of 99 cases of 2019 novel coronavirus pneumonia in Wuhan, China: a descriptive study. Lancet. 2020; 395(10223):507-13.

2. Bai $Y$, Yao L, Wei $T$, Tian F, Jin DY, Chen $L$, et al. Presumed asymptomatic carrier transmission of COVID-19. JAMA. 2020; 323(14):1406-7.

3. Li B, Yang J, Zhao F, Zhi L, Wang X, Liu L, et al. Prevalence and impact of cardiovascular metabolic diseases on COVID-19 in China. Clin Res Cardiol. 2020;109:531-8.

4. American Diabetes Association. 3. Comprehensive medical evaluation and assessment of comorbidities. Diabetes Care. 2020;40(Suppl 1): S25-S32.

5. Akbar $\mathrm{DH}$. Bacterial pneumonia: comparison between diabetics and non-diabetics. Acta Diabetol. 2001;38(2):77-82

6. Yang X, Yu Y, Xu J, Shu H, Liu H, Wu Y, et al. Clinical course and outcomes of critically ill patients with SARS-CoV-2 pneumonia in Wuhan, China: a single-centered, retrospective, observational study. Lancet Respir Med.2020;5(8):475-81.

7. Hill MA, Mantzoros C, Sowers JR. Commentary: COVID-19 in patients with diabetes. Metabolism. 2020;107:154217.

8. Klonoff DC, Umpierrez GE. COVID-19 in patients with diabetes: risk factors that increase morbidity. Metabolism. 2020 Apr 7:154224. doi: 10.1016/j.metabol.2020.154224. Online ahead of print

9. Hussain A, Bhowmik B, do Vale Moreira NC. COVID-19 and diabetes: Knowledge in progress. Diabetes Res Clin Pract. 2020; Apr 162:108142.

10. Wang W, Lu J, Gu W, Zhang Y, Liu J, Ning G. Care for diabetes with COVID-19: Advice from China. J Diabetes. 2020;12(5):417-9.

11. Huang C, Wang Y, Li X, Ren L, Zhao J, Hu Y, et al. Clinical features of patients infected with 2019 novel coronavirus in Wuhan, China. Lancet. 2020;395(10223):497-506.

12. Awadhesh K, Singha R, Guptab A, Ghoshb AM. Prevalence, pathophysiology, prognosis and practical considerations Diabetes in COVID-19. Diabetes Metab Syndr. 2020;14(4):303-10.

13. Vaninov N. In the eye of the COVID-19 cytokine storm. Nat Rev Immuno. 2020;20:277.

14. Buonaguro FM, Ascierto PA, Buonaguro L, Morse GD, Tornesello ML, Puzanov I, et al. COVID-19: a paradigm change. SSRN Electronic Journal. $2020 ; 3561224$
15. Chen $C$, Zhang XR, Ju ZY, He WF. [Advances in the research of cytokine storm mechanism induced by corona virus disease 2019 and the corresponding immunotherapies]. Zhonghua Shao Shang Za Zhi. 2020;36:E005

16. Rojas-Martínez R, Basto-Abreu A, Aguilar-Salinas CA, Zárate-Rojas E, ViIlalpando S, Barrientos-Gutiérrez T. Prevalence of previously diagnosed diabetes mellitus in Mexico. Salud Pública de Méx. 2018;60(3):224-32.

17. Protocolo de atención para COVID-19 (SARS-CoV-2) de la Sociedad Mexicana de Medicina de Emergencias [Internet]. México: Sociedad Mexicana de Medicina de Emergencia, A.C. [09 mayo 2020]. Disponible en: https://www.flasog.org/static/COVID-19/GuiaCOVID19SMME.pdf

18. George CM, Byun A, Howard-Thompson A. New injectable agents for the treatment of type 2 diabetes part 1 - Injectable insulins. Am J Med. 2018;131(7):752-4.

19. Recomendaciones SMNE COVID-19 [Internet]. México: Sociedad Mexicana de Nutrición y Endocrinología [09 mayo 2020]. Disponible en: https:// endocrinologia.org.mx/smne-covid-19.php

20. Saisho Y. Metformin and inflammation: Its potential beyond glucose-lowering effect. Endocr Metab Immune Disord Drug Targets. 2015;15(3):196-205.

21. Hitchings AW, Lai D, Jones PW, Baker EH. Effects of metformin on serum Creactive protein and interleukin- 6 concentration in acute exacerbations of chronic obstructive pulmonary disease: A multi-centre, randomized, double-blind, placebo-controlled trial. Am J Respir Crit Care Med. 2020;201:A3966.

22. Ho TW, Huang CT, Tsai YJ, Lien ASY, Lai F, Yu CJ. Metformin use mitigates the adverse prognostic effect of diabetes mellitus in chronic obstructive pulmonary disease. Respir Res. 2019;20(1):69.

23. Maddaloni E, Buzzetti R. Covid-19 and diabetes mellitus: unveiling the interaction of two pandemics. Diabetes Metab Res Rev. 2020 Mar 31;e33213321. doi: 10.1002/dmrr.3321. Online ahead of print.

24. Rajeshkumar NV, Yabuuchi S, Pai SG, Maitra A, Hidalgo M, Dang CV. Fatal toxicity of chloroquine or hydroxychloroquine with metformin in mice. bioRxiv. 2020April 04. doi: https://doi.org/10.1101/2020.03.31.018556. Online ahead of print.

25. Pal R, Bhadada SK. Should anti-diabetic medications be reconsidered amid COVID-19 pandemic? Diabetes Res Clin Pract. 2020 Ap 10;163:108146. doi: 10.1016/j.diabres.2020.108146. Online ahead of print

26. Drucker DJ. Coronavirus Infections and type 2 diabetes-shared pathways with therapeutic implications. Endocr Rev. 2020;41(3):bnaa011.

27. Gorricho J, Garjón J, Alonso A, Celaya MC, Saiz LC, Erviti J, et al. Use of oral antidiabetic agents and risk of community-acquired pneumonia: a nested case-control study. Br J Clin Pharmacol. 2017;83(9):2034-44.

28. Pitocco D, Tartaglione L, Viti L, Di Leo M, Pontecorvi A, Caputo S. SARS CoV-2 and DPP4 inhibition: is it time to pray for Janus Bifrons? Diabetes Res Clin Pract. 2020 Apr 23;163:108162. doi: 10.1016/j.diabres.2020.108162. Online ahead of print.

29. lacobellis G. Local and systemic effects of the multifaceted epicardia adipose tissue depot. Nat Rev Endocrinol. 2015;11(6):363-7.

30. Kulcsar KA, Coleman CM, Beck SE, Frieman MB. Comorbid diabetes results in immune dysregulation and enhanced disease severity following MERS-CoV infection. JCI Insight. 2019;4(20):e131774.

31. Gentile S, Strollo F, Ceriello A. COVID-19 infection in Italian people with diabetes: lessons learned for our future (an experience to be used). Diabetes Res Clin Pract. 2020 Apr;162:108137.

32. Singh AK, Gupta R, Ghosh A, Misra A. Diabetes in COVID-19: Prevalence, pathophysiology, prognosis, and practical considerations. Diabetes Metab Syndr. 2020;14(4):303-10.

33. Zhang W, Li C, Liu B, Wu R, Zou N, Xu YZ, et al. Pioglitazone upregulates hepatic angiotensin converting enzyme 2 expression in rats with steatohepatitis. Ann Hepatol. 2013;12(6):892-900.

34. Patti AM, Rizvi AA, Giglio RV, Stoian AP, Ligi D, Mannello F. Impact of glucose-lowering medications on cardiovascular and metabolic risk in type 2 diabetes. J Clin Med. 2020;9(4):912.

35. Zhou J, Tan J. Diabetes patients with COVID-19 need better blood glucose management in Wuhan, China. Metabolism. 2020;107:154216.

36. Khunti S, Khunti N, Seidu S, Khunti K. Therapeutic uncertainties in people with cardiometabolic diseases and severe acute respiratory syndrome coronavirus 2 (SARS-CoV-2 or COVID-19). Diabetes Obes Metab. 2020 Apr 21. doi: 10.1111/dom.14062. Online ahead of print.

37. American Diabetes Association. 15. Diabetes care in the hospital: Standards of medical care in diabetes-2020. Diabetes Care. 2020;43(Suppl 1):S193-S202.

38. FAQs on Home-use Blood Glucose Meters Utilized Within Hospitals During the COVID-19 Pandemic [Internet]. EE.UU.: Fodd and Drug Administration [fecha de actualización: 13 de abril de 2020]. Disponible en: https://www.fda. $\mathrm{gov} / \mathrm{medical}$-devices/blood-glucose-monitoring-devices/faqs-home-useblood-glucose-meters-utilized-within-hospitals-during-covid-19-pandemic

39. Thompson BM, Mackey PA, Cook CB. Management of insulin pumps in hospitalized patients. En: Matfin G, editor. Endocrine and Metabolic Medical Emergencies: A Clinician's Guide, Second Edition. John Wiley \& Sons; 2018. pp. 571-83. 